\title{
Estimation of particle size distribution of nanoparticles from electrical characteristics
}

\author{
ARNAB GANGOPADHYAY*, ADITI SARKAR and A SARKAR \\ Department of Physics, Bijoy Krishna Girls' College, Howrah 711101, India \\ *Author for correspondence (agangulyphysics@gmail.com)
}

MS received 9 January 2016; accepted 13 August 2017; published online 2 February 2018

\begin{abstract}
An indirect method of estimation of size distribution of nanoparticles in a nanocomposite is proposed in this paper. The present approach exploits DC electrical current-voltage characteristics (CVC) of ZnO nanocomposite specimen in bio-polymer background. The nature of DC CVC is found to be oscillatory with respect to applied voltage. The nature of CVC is a consequence of Coulomb blockade (CB) phenomena of electrical conduction through a tiny nanoparticle. Considering the $\mathrm{ZnO}$ nanocomposites to be spherical, Coulomb-blockade model of quantum dot is applied here. The size distribution of particle is estimated from that model and compared with the results obtained from AFM and XRD analyses. The results from CVC are found to be consistent with these conventional microscopic results.
\end{abstract}

Keywords. Electrical characteristic; Coulomb blockade; quantum dot.

\section{Introduction}

Nanocomposites (NC) occupied a leading role in the field of science, technology and material science. The physical and chemical properties of a material are largely dependent on the structure and the constituents of it. The chemical activities and physical properties of a material changes significantly with the decrease in size of the constituent clusters, especially, when it is of the order of the nanorange. It is desirable that estimation of particle size of a sample may be obtained by analysing its physical properties. Many novel properties are found to emerge when surface to volume ratio of the clusters increases [1]. These properties are widely different from their bulk correspondence. Study of the physical and chemical properties of the nanomaterials are important and such properties chiefly depend on the size of the particles. Generally X-ray diffraction (XRD), atomic force microscopy (AFM) and other high resolution microscopy techniques are used to extract information of the sizes of the constituent particles. Although these conventional methods are appropriate for determining the particle size, they are expensive and generally require a long time. In this work, a new technique is proposed to determine the sizes of nanoparticles (NPs) by electrical characterization. Eventually, this new process is faster than other conventional processes.

In this work, we use the bottom-up process to prepare NC. The sizes of these NPs are distributed over a certain range instead of a definite size [2].

The longitudinal DC electrical conduction through the developed system is studied experimentally. Quantum effect plays dominant role in electrical transport through the developed $\mathrm{NC}$ even at room temperature (RT). The DC current-voltage characteristics (CVC) shows an oscillation in the current. Regular oscillation was recorded in d.c. $I-V$ (current-voltage) or $g-V$ (conductance-voltage) characteristics of many nanosystems. In some earlier work, Sarkar and his co-wokers [3-5] have observed such regular oscillation in nanosystems (e.g., $\mathrm{Cr}_{2} \mathrm{O}_{3}$ in acacia background [3]; $\mathrm{ZnS}, \mathrm{ZnO}$ in Styrofoam background; Co-doped $\mathrm{ZnO}$ [4]; $\mathrm{Cr}_{1-x} \mathrm{~V}_{x} \mathrm{~S}_{2}$ in polymeric Gum acacia background [5]). Overall nature of the observed characteristics is marked as fingerprint of the existence nanocluster in the background matrix. The objective of the present work is to develop a nanocharacterization technique by exploiting oscillatory d.c. $I-V$ characteristics of nanosystem.

The nature of the oscillatory currents are explained by the Coulomb blockade (CB) model of the quantum dot and the radius of the NPs are found by considering the NPs as quantum dots. The detailed experimental results, theoretical considerations, measurement of size of the NPs and the comparison with standard methods are discussed in the following sections and subsections.

\section{Sample preparation and measurement}

In general, NPs may be prepared in two ways [6]. They can either be prepared by grinding bulk materials or by chemical synthesis route. The first one is known as top-down process, whereas the later is known as the bottom-up process. Since the freshly prepared NPs are hyperactive in the 
bottom-up process, a capping material is required to prevent the formation of large cluster of NPs. Prepared NPs are randomly distributed throughout the bulk of the capping materials. This bottom-up route is cheaper than top-down route. $\mathrm{ZnO} \mathrm{NC}$ was synthesized following sol-gel route at $105^{\circ} \mathrm{C}$. Zinc oxide $(\mathrm{ZnO}) \mathrm{NC}$ was prepared from analytical grade zinc acetate, $\left(\mathrm{Zn}\left(\mathrm{CH}_{3} \mathrm{COO}\right)_{2}\right)$ from $\mathrm{CDH}$, India $\mathrm{Ltd}$., and analytical grade gum acacia from E. Merck Ltd. The later is a good candidate for capping agent due to high thermal and chemical stabilities [7]. $\left(\mathrm{Zn}\left(\mathrm{CH}_{3} \mathrm{COO}\right)_{2}\right)$ weighing $1.0 \mathrm{~g}$ was dissolved in $50 \mathrm{ml}$ distilled water. Gum acacia powder weighing $3.0 \mathrm{~g}$ was dissolved in $50 \mathrm{ml}$ hot-distilled water separately. These two solutions were mixed in 1:2 volumetric ratio to get $75 \mathrm{ml}$ final solution. This solution was refluxed continuously at temperature about $105^{\circ} \mathrm{C}$ for $15 \mathrm{~h}$. Final product was cast to form experimental pellets with thickness and diameter of 0.5 and $12 \mathrm{~mm}$, respectively, after adequate drying. The prepared sample was marked as 'A'. A second sample was prepared by the same process, but with the concentration of $\mathrm{Zn}\left(\mathrm{CH}_{3} \mathrm{COO}\right)_{2}$ doubled. This sample was marked as ' $\mathrm{B}$ '.

\section{Experimental}

The sample was sandwiched between the two flat polished $\mathrm{Cu}$ electrodes. A thin layer of silver paste was used between the NC sample and the electrodes to achieve firm electrical contact. The instrumental setup is described in figure 1. CVC of the developed NCs was measured with respect to applied voltage by PC interfaced Keithley 2400 (USA) source meter at RT to have information about the charge transport phenomena. Two samples were measured separately in a voltage range between 0 and $2.5 \mathrm{~V}$ in $30 \mathrm{mV}$ step. AFM measurement was carried out with Cervantes Full Mode AFM System from Nanotech and XRD measurement was carried out with Rigaku Miniflex XRD System (Japan) using CuK line in $20 \mathrm{kV}$.

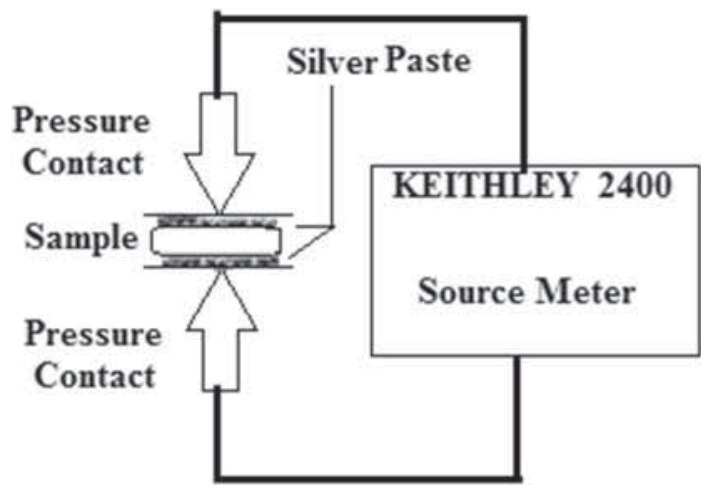

Figure 1. Experimental setup used for measuring $I-V$ characteristics of NC system.

\section{Results and discussion}

XRD of the sample is shown in figure 2. The particle size from XRD was estimated using Debye-Scherrer formula [8]. The particle size thus obtained is given in table 1. It is clear that the sample contains NP with size of the order of 14-30 nm. This particular bottom-up process can be used to develop NC.

The DC response current of two samples were measured with voltage in step of $30 \mathrm{mV}$ at RT. The variation of current with respect to voltage is shown in figure 3 . The measured current was found to oscillate with respect to applied voltage. The CVC is found to be oscillatory in this particular sample due to the confinement of $\mathrm{ZnO}$ NPs in the background polymeric matrix. The nanoimplanted material is investigated mostly through its DC characteristics. The experimental results reveal that the nature of $\mathrm{CVC}$ becomes oscillatory after inclusion of NPs in the gum acacia matrix. Thus, the oscillations are supposed to carry information about size distribution

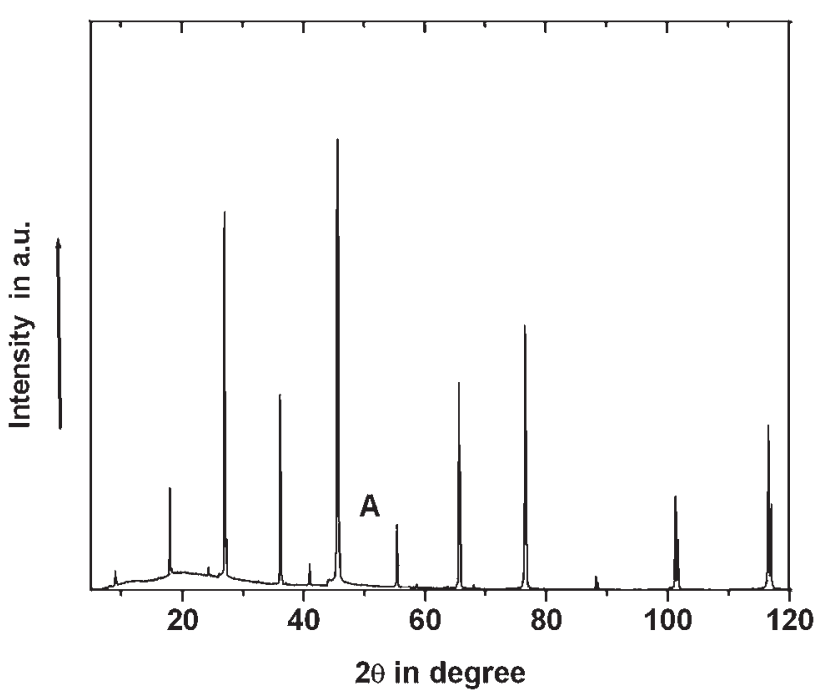

Figure 2. XRD of sample A.

Table 1. Particle size and corresponding peak positions as found from XRD analysis.

\begin{tabular}{lc}
\hline Peak position & Particle size $(\mathrm{nm})$ \\
\hline 17.87 & 14.484 \\
26.9 & 14.759 \\
36.08 & 15.165 \\
45.53 & 15.731 \\
55.31 & 16.499 \\
65.57 & 17.543 \\
76.46 & 18.986 \\
101.24 & 22.218 \\
116.48 & 26.804 \\
\hline
\end{tabular}



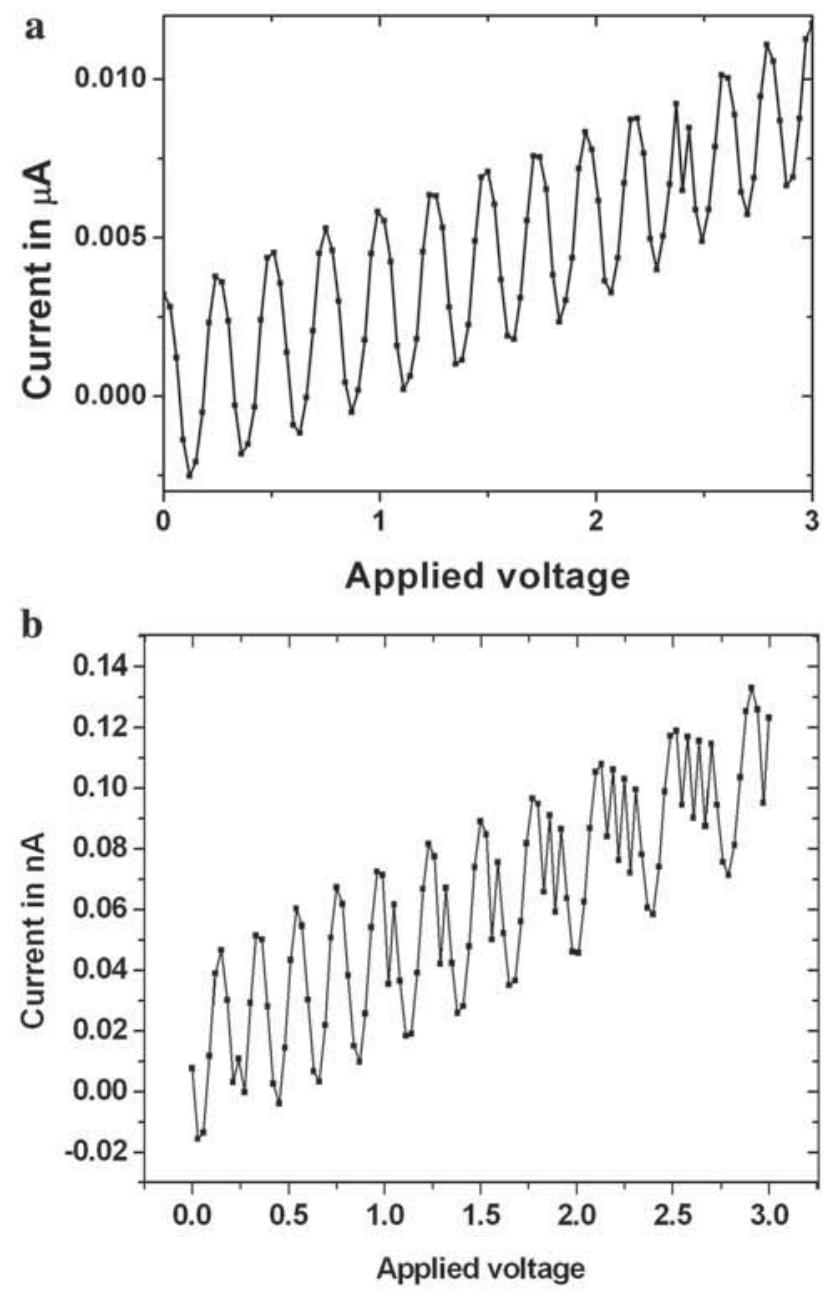

Figure 3. DC CVC of samples A and B.

of nanoparticles. The slope of CVC is due to ohmic resistivity of background material mostly, but this does not affect the analysis for estimation of sizes of the implanted NPs. The $30 \mathrm{mV}$ voltage step is an appropriate choice since autocorrelation in measuring the data is the highest at this voltage step when compared to other voltage steps. CVC becomes noisy and random for smaller and higher voltage steps. Below $30 \mathrm{mV}$, the step size is comparable to the thermal excitation at RT $(\sim 25 \mathrm{meV})$.

The interval of voltage after which the oscillation of measured current is periodic can be termed as the period of the oscillation. It is clear from figure 3 that the nature of oscillation is different for two different samples. Thus, the periods of oscillation of CVC carry most important information about the size distribution of the particle. The periods of oscillation and corresponding amplitudes of these oscillations were obtained by numerical Fourier transform (FT) [9] of the experimentally obtained data. This numerical FT of CVC is shown in figure 4. Existences of multiple peaks in figure 4 indicate that the response current of a sample oscillates in different frequencies. Here, it is also
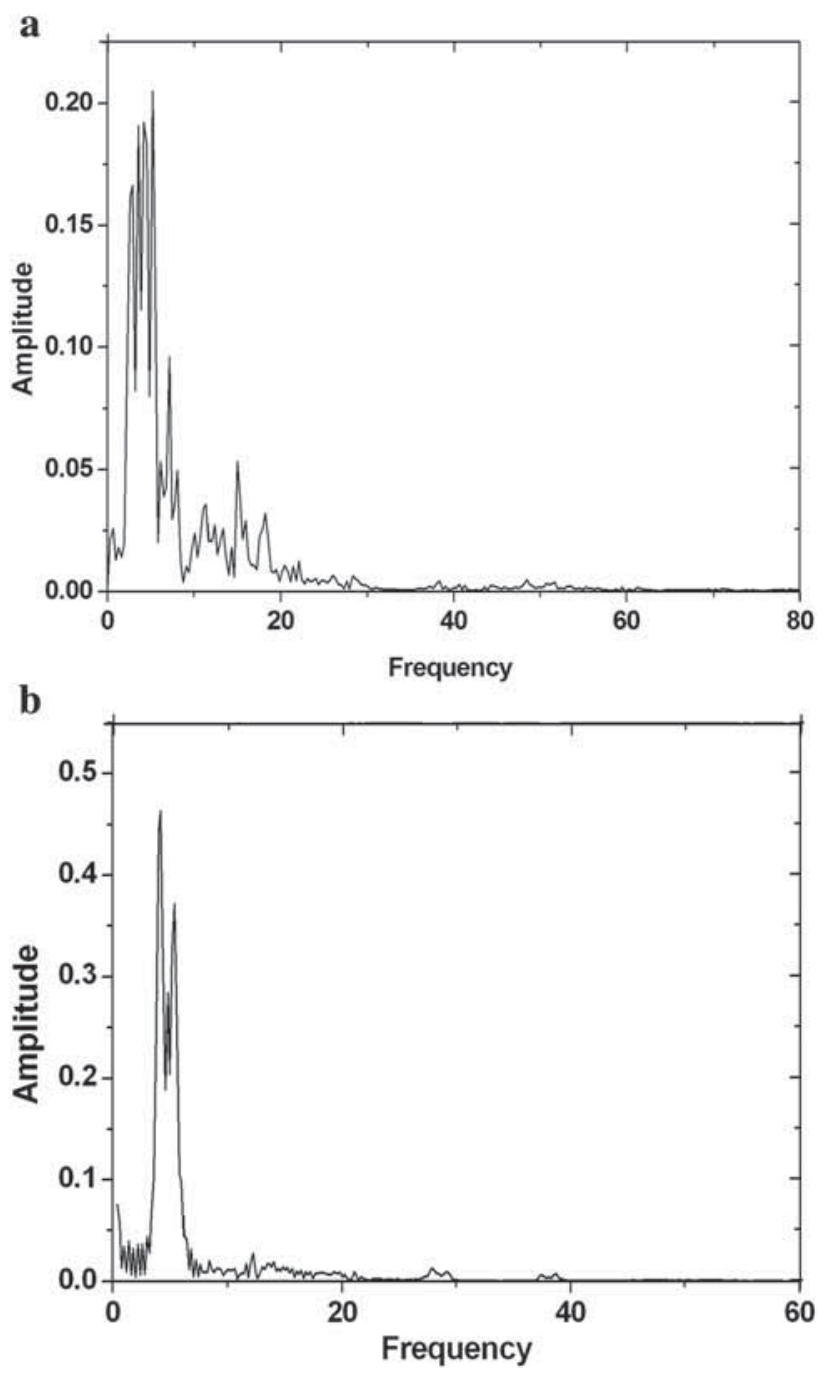

Figure 4. Numerical FT of DC CVC of samples A and B. Graphs correspond to samples A and B.

assumed that the amplitude of a particular harmonic is proportional to number density of NP corresponding to that harmonic.

The nature of CVC may be attributed to quantum effect. The existence of oscillation and discontinuities in CVC are almost Coulomb charging like steps [10]. The mentioned oscillatory nature of electrical conduction through nanocontact may be explained [11-14] in terms of CB model. Previous works [15, 16] already demonstrated that the $\mathrm{CB}$ effect can be observed in RT.

In CB model, a quantum dot and its connecting leads can be represented by an electrical equivalent circuit $[10,17]$, shown in figure 5. The nanoclusters in the sample under study are very close to each other and they can be regarded as quantum dots.

The roles of the leads and gates of a particular NP, i.e., one quantum dot, are carried by neighbouring NPs of the system. The conducting electrical path in the sample is completed via 


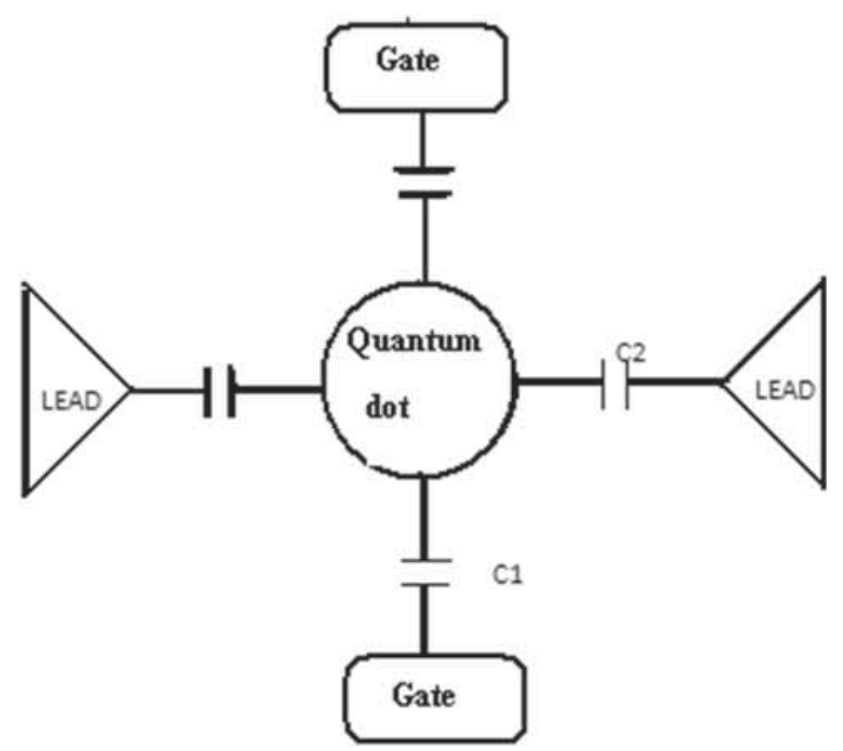

Figure 5. Electrically equivalent model circuit of quantum dot.

randomly distributed NPs and the background material, Gum acacia [18-22]. The back ground capping media, Gum acacia is $97 \%$ ionic, 3\% electronic conductor [7]. Acacia has a little effect in electronic conduction and it may be considered as a dielectric material. This offers a small Ohmic background on which the oscillatory nature of CVC of $\mathrm{NC}$ is superposed, as shown in figure 3. Nanoclusters are embedded in the background polymer material which has to posses some criterion to sustain the oscillations of current in DC CVC. The background material must be soft ionic polymer in nature with low electrical activation potential and low electronic conductivity. The background material used in present work is Acacia arabica. Its electro-thermal activation energy and electronic conductivity are estimated to be $0.17 \mathrm{eV}$ and $1.5 \times 10^{-6} \mathrm{~S} \mathrm{~m}^{-1}$, respectively [7]. Quantum effect supersedes thermal fluctuation in these circumstances and CB effects are detectable. Previous works $[5,23,24]$ also support the presence of oscillatory nature of DC CVC of NCs in Gum acacia background.

However, according to $\mathrm{CB}$ model, a nanodot of definite size causes the response current to oscillate at a definite frequency [25-27] and the period of oscillation is given by refs. [28-33].

$$
\omega=e / C
$$

where $e$ is the electronic charge and $C$ is the average capacitance of the connecting leads and the quantum dot. As it is assumed that particles are spherical, the capacitance $C$ of the CB model can be replaced by

$$
C=4 \pi \varepsilon R
$$
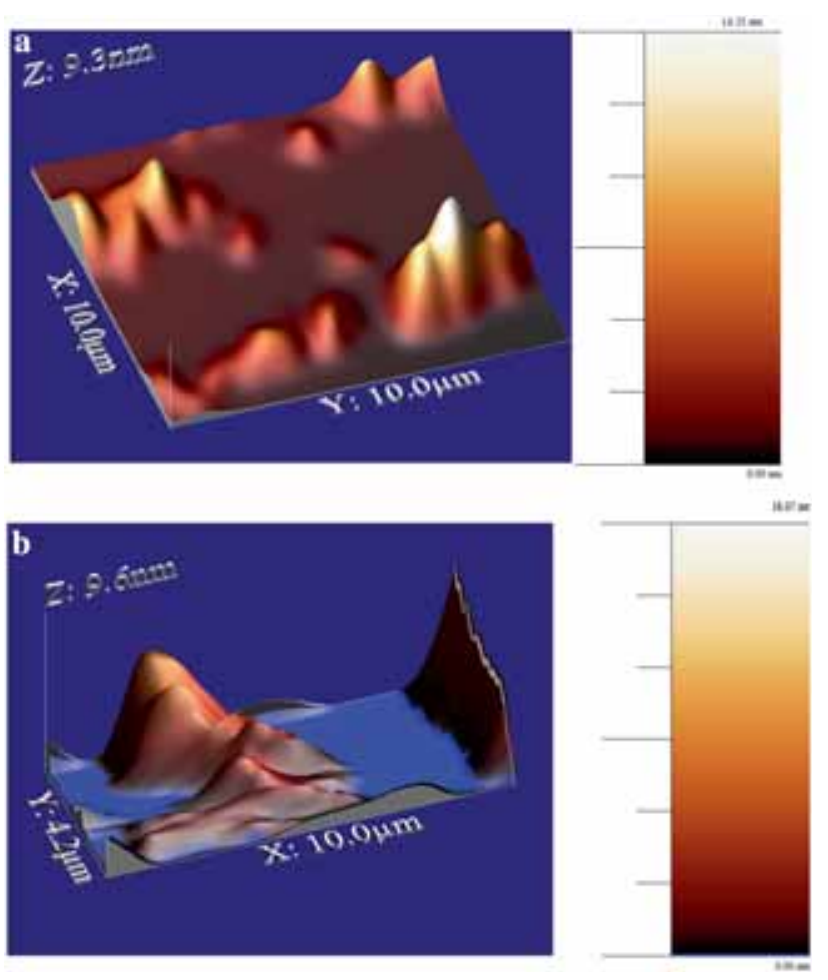

Figure 6. AFM microgram image of samples A and B.

where $R$ is the radius of the quantum dot and $\varepsilon$ is the permittivity of the acacia base. Following Gerasimov et al [34] and Wasshuber [35,36], capacitance of nanosize material may be expressed in an infinite series. The first term of the series may be given by equation (2). The convergence of the series found to be dependent on separation distance between NPs. It has been found that the series converges to $4 \pi \varepsilon R$ at separation distance of the order of $10 \mathrm{~nm}$. In the present study, the separation distance is $>10 \mathrm{~nm}$ and equation (2) is a legitimate choice. The measured permittivity of the acacia is $14.16 \times 10^{-12} \mathrm{~F} \mathrm{~m}^{-1}$. Thus

$$
R=e /(4 \pi \varepsilon \omega)
$$

Hence, the size of the quantum dot i.e., the size of the particle can be found from the information of $\omega$ using figure 5 and equation (3). Moreover, it is observed from figure 5 that period of current oscillation $\omega$ is distributed over a range. Hence, according to equation (2), $R$ is also distributed over a range.

The AFM study was also carried on the samples A and B. The micrograms thus obtained are shown in figure 6 for first and second samples, respectively. The particle distribution in two samples is estimated with the software WXFM 5.0 associated with AFM setup. The topological features in AFM data result are due to the NPs. The base polymeric matrix exhibits no topological features [37]. Topological features appear in the presence of nanocluster with size 

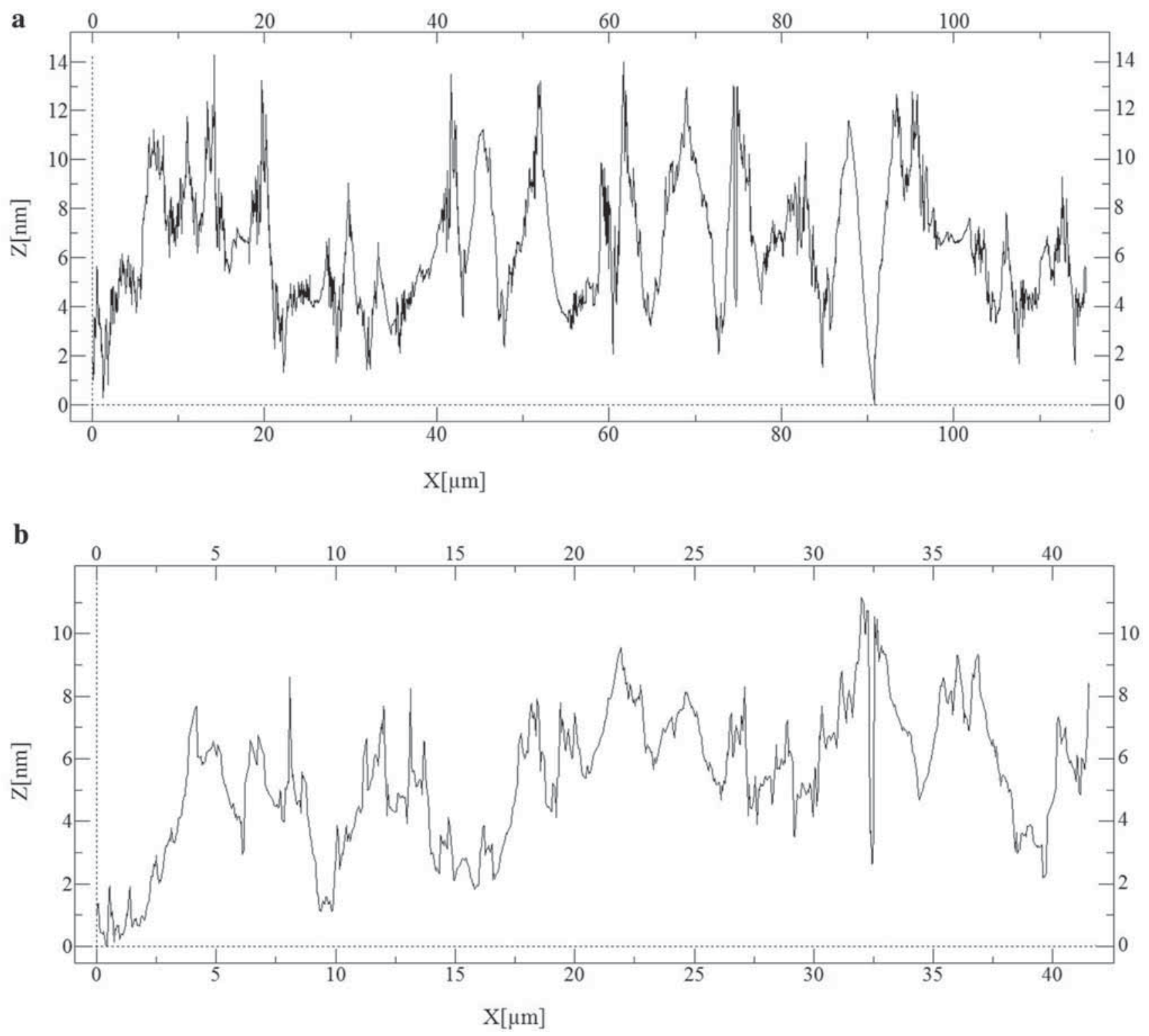

Figure 7. Z-profile of AFM microgram image for samples A and B.

$\sim 5 \mathrm{~nm}$ or above in the base matrix. The profile height in AFM microgram is related to the particle size as shown in figure 7.

Figure 8 shows the comparison of distribution of particle size by $\mathrm{CB}$ model (continuous line) with AFM study (dotted line) for both samples A and B, respectively. Both figures demonstrate the number of particles in $x$-axis and corresponding bin size in $x$-axis. The figures are associated with error bars. Estimation of error in accumulated data is carried out by the software MS ORIGIN. The size of a nanocluster is identified as the full-width at half-maxima (FWHM) of the peaks appeared in the particle size distribution curve extracted from AFT analysis (figure 7). Evaluation of FWHM of each peak is done by fitting Gaussian curve using Ms
Origin and the error in the curve fitting is provided by the software. Error estimation in determination of particle sizes from DC CVC method is same as above, but applied to peak position.

The distribution of particle size obtained from AFM micrograph analysis is well-consistent with that obtained from the new CVC method. The distribution of particle size from XRD data is also in good agreement with that obtained from the other two methods. It is clear from the figure that the distribution of size for two samples A and B are not same. The result is consistent since the samples were prepared under two different concentrations. Higher concentration leads to higher reaction rate. The different reaction rate results in differences in clustering size. 

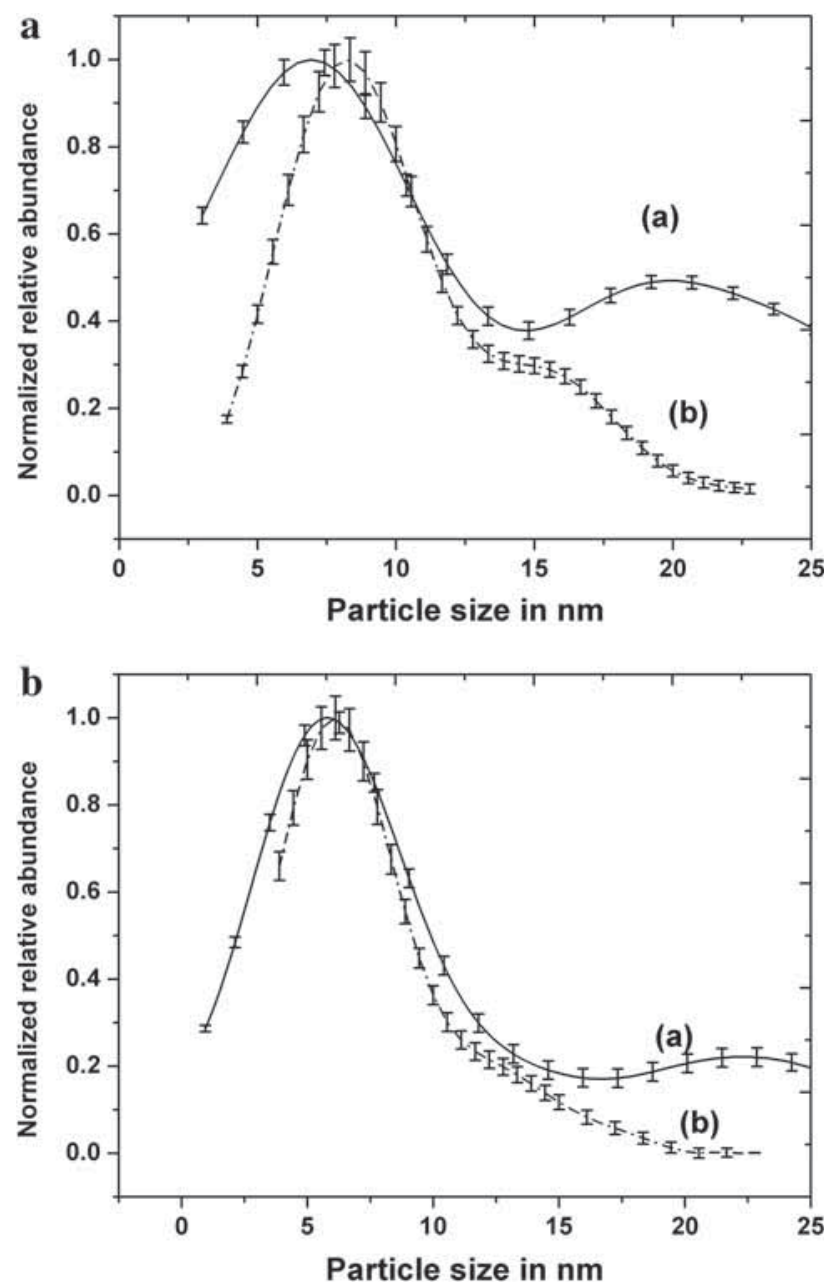

Figure 8. Particle size distribution of samples. Graphs ' $a$ ' and 'b' correspond to samples A and B, respectively. (a) Size distribution found from CVC and (b) AFM micrograph of size distribution.

\section{Conclusions}

In summary, it is shown that the oscillations in CVC of $\mathrm{NC}$ arise from $\mathrm{CB}$ effect of constituent nanoclusters of NCs. This nature of the DC CVC is detectable for nanosamples even at the RT. The different Fourier components of the oscillatory current originate from the differences in sizes of the clusters. The amplitude of a particular Fourier component is proportional to number density of NP corresponding to that component. The particle size distribution determined from DC CVC agrees well with the results obtained from conventional AFM and XRD experiments.

\section{Acknowledgements}

Arnab Gangopadhyay is thankful to UGC, New Delhi, for offering SRF (Fellowship no. F.11-10/2011 (SA-I)). Aditi Sarkar is thankful to CSIR, New Delhi (Fellowship no: 08/456(0002)/2010-EMR-I), for offering SRF. Sarkar is thankful to UGC, New Delhi, for UGC-CPE grant. We specially thank Prof Jacob of IIT KGP, for providing AFM facility.

\section{References}

[1] Cao G and Wang X 2011 Nano structures and nano materials, synthesis, properties and applications (US: World Scientific)

[2] Wang Z 2000 Characterization of nano phase materials (Federal Republic of Germany: Wiley-VCH Verlag $\mathrm{GmbH}$ Weinheim)

[3] Gupta N, Mallik H and Sarkar A 2005 J. Metastab. Nanocrystal. Mater. 23335

[4] Barman M, Paul S and Sarkar 2013 Adv. Appl. Sci. Res. 4343

[5] Gangopadhyay A, Sarkar A and Sarkar A 2013 Proc. Adv. Mater. Res. 665233

[6] Whitesides G, Kriebel J and Mayers B 2005 Self assembly and nano structured materials (US: Springer)

[7] Kalia S and L Averous (eds) 2011 Biomedical and environmental application. In Biopolymers (Wiley, NJ, USA: Scrivener Publishing)

[8] Cullity B D 1956 Elements of X-ray diffraction (Reading, USA: Addison-Wesley Publishing Company Inc.)

[9] Arfken G B and Weber H J 2005 Mathematical methods for physicists (MA, USA: Elsevier Academic Press)

[10] Taylor P L and Heinonen O 2002 A quantum approach to condensed matter physics (Cambridge, UK: Cambridge University Press)

[11] Likharev K K 1988 C R Acad. Sci. Paris 32144

[12] Averin D V and Likharev K K 1991 In Mesoscopic phenomena in solids (eds) B Altshuler, P A Lee and R A Webb (Amsterdam, The Netherlands: Elsevier)

[13] Kuzmin L S and Likharev K K 1987 JETP Lett. 45389

[14] Lee K and Zhong Z 2015 Bull. Am. Phys. Soc. 601

[15] Kubatkin S E, Danilov A V, Bogdanov A L, Olin H and Claeson T 1998 Appl. Phys. Lett. 733604

[16] Aceves Z Y M, Wang F, Carrillo J, Kiebach R and Monfil K 2011 Phys. E 621118

[17] Mullen K, Ben-Jacob E, Jaklevic R C and Schuss Z 1988 Phys. Rev. B 3798

[18] Beenakker C W J 1991 Phys. Rev. B 441646

[19] Scott-Thomas J H F, Field S B, Kastner M A, Smith H I and Antoniadis D A 1989 Phys. Rev. Lett. 62583

[20] Field S B, Kastner M A, Meirav U, Scott-Thomas J H F, Antoniadis D A, Smith H I et al 1990 Phys. Rev. B 423523

[21] de Graaf C, Caro J, Radelaar S, Lauer V and Heyers K 1991 Phys. Rev. B 449072

[22] Staring A A M, van Houten H, Beenakker C W J and Foxon C T 1992 In High magnetic fields in semiconductor physics (eds) G Landwehr III (Berlin: Springer)

[23] Gangopadhyay A, Sarkar A and Sarkar A 2011 Adv. Appl. Sci. Res. 2149

[24] Gangopadhyay A, Sarkar A and Sarkar A 2011 Asian J. Chem. 235581

[25] Gorter C J 1951 Physica 17777

[26] Lambeir R, van Itterbeek A and van den Berg G J 1950 Physica 16907 
[27] Mostovech N and Vodar B 1950 C R Acad. Sci. Paris 230 934

[28] Fulton T A and Dolan G J 1987 Phys. Rev. Lett. 59109

[29] Amman M, Mullen K and Ben-Jacob E 1989 J. Appl. Phys. 65 339

[30] Glazman L I and Shekhter R I 1989 J. Phys. Condens. Matter 15811

[31] Chutia P and Kumar A 2015 Polym. Eng. Sci. 55995

[32] Gu H et al 2014 Polymer 554405
[33] Yu G-F, Xu Y, Zhang H-D and Long Y-Z 2015 Nanoscale Res. Lett. 101

[34] Gerasimov Y S, Shorokhov V V, Maresov A G, Soldatov E S and Snigirev O V 2011 J. Commun. Technol. Electron. 561483

[35] Wasshuber C 1998 Computational microelectronics (New York: Springer Verlag)

[36] Bischo D, Eich M, Varlet A, Simonet P, Ihn T and Ensslin K 2015 Phys. Rev. B 91115441

[37] Mullick H and Sarkar A 2002 Mater. Sci. Eng. C 20215 